\title{
Yang-Baxter Equations and Intermediate Long Wave Hierarchies
}

\author{
C. Morosi ${ }^{1}$ and G. Tondo ${ }^{2}$ \\ ${ }^{1}$ Dipartimento di Matematica, Università di Perugia, Via Vanvitelli, I-06100 Perugia, Italy \\ ${ }^{2}$ Dottorato in Matematica, Università di Milano, Via Saldini 50, I-20133 Milano, Italy
}

\begin{abstract}
In the framework of the GN manifold approach, the algebraic structures of two representations of the ILW hierarchy are investigated. It is shown that this hierarchy can be obtained by different realizations of two abstract structures, strictly related with the Yang-Baxter equations. A new reduction theorem for the second representation of ILW is also formulated.
\end{abstract}

\section{Introduction}

It is well-known that the differential non-linear evolution equations (DNEE) in $(1+1)$ dimensions integrable by the Inverse Spectral Transform are bihamiltonian, with an infinite hierarchy of constants of the motions in involution [1]. More recently, this peculiar property has been pointed out also for many DNEE's in $(2+1)$ dimensions $([1,2,3]$ and references therein).

On the other hand, there are some integro-differential evolution equations (INEE) in $(1+1)$ dimensions, such as the Benjamin-Ono (BO) [4] and the Intermediate Long Wave equation (ILW) $[5,6,7]$, which algebraic and analytical features quite similar to those of DNEE's in $(2+1)$ dimensions. In fact, they have been shown to be bihamiltonian $[8,9]$ only in the framework of the so-called extended formalism, which was introduced just for DNEE's in $(2+1)$ dimensions.

In this paper we investigate the algebraic structure of the ILW hierarchy and its deep relation with the Yang-Baxter equations $[10,11]$. We make use of the GN manifold approach [3]: in this framework, by introducing a recursion (Nijenhuis) operator $\mathrm{N}$ and a symmetry group $\mathrm{G}$ of $\mathrm{N}$ one can construct the most part of the integrable bihamiltonian DNEE's in $(2+1)$ dimensions $[12,13]$. The evolution equations are explicitly obtained by a suitable reduction of the Lenard bicomplex, i.e. a two-indices family of vector fields in involution making a Kac-Moody type algebra. Our first result is to show that the so-called two representations [9] of the ILW hierarchy correspond, roughly speaking, to particular solutions of two remarkable equations, the modified Yang-Baxter (mYB) equation and the Yang-Baxter (YB) equation. Indeed, we are able to construct two classes of GN manifolds and Lenard bicomplexes for any solution of these equations. Then, by choosing two particular solutions, we specialize the two structures and prove two reduction 
theorems for the corresponding Lenard bicomplexes. While the first one is only a slight extension of the theorem given in [3] (which was successfully used for the ZS-AKNS and Veselov-Novikov hierarchies), the second one is altogether different and provides a new reduction technique.

Finally, the two Lenard bicomplexes are realized on a suitable algebra, giving rise to two representations of the ILW hierarchies both in $(1+1)$ and $(2+1)$ dimensions. We remark that in this framework the extension to $(2+1)$ dimensions is quite natural, corresponding to different choices of some constant parameter in the abstract algebra underlying the GN manifold.

The paper is organized as follows. A short review of the GN manifold and of the Lenard bicomplex is given in Sect. 1, only to recall the main definitions and notations to be used throughout the paper. In Sects. 2, 3 we perform the construction and the reduction of a GN manifold on an associative algebra, based on the solution of the $\mathrm{mYB}$ equation. As a particular realization of such a reduction, the ILW hierarchy is obtained in Sect. 4. Another GN manifold, corresponding to the solutions of the YB equation, is constructed and reduced (by a new technique) in Sects. 5, 6. As a particular realization, it gives rise to a hierarchy of INEE's which turns out to be equivalent to the previous one.

\section{GN Manifolds and Lenard Bicomplexes}

In this section we introduce the GN manifold and the Lenard bicomplex: they are the geometric structures on which the construction of the hierarchies of evolution equations is based.

On a differentiable manifold $M$, a linear mapping $N: \mathscr{X}(M) \rightarrow \mathscr{X}(M)$ defines a Nijenhuis tensor field if it fulfills the realization

$$
[N \varphi, N \psi]-N[N \varphi, \psi]-N[\varphi, N \psi]+N^{2}[\varphi, \psi]=0
$$

for any pair of vector fields $\varphi, \psi \in \mathscr{X}(M),[$,$] being the commutator of vector fields.$ A Lie group $G$, acting on $M$ by $\Phi: G \times M \rightarrow M$, is said to be a symmetry group for $N$ if the infinitesimal generator of $\Phi, X: \mathscr{G} \rightarrow \mathscr{X}(M)$, is such that the vector fields

$$
\varphi_{a}(u):=X_{u} a \quad(u \in M, a \in \mathscr{G})
$$

are symmetries of $N$, i.e. the Lie derivative of $N$ with respect to $\varphi_{a}$ vanishes:

$$
L_{\varphi_{a}}(N)=0 .
$$

Then it can be proved that the vector fields $\varphi_{a}^{j}:=N^{j} X a(a \in \mathscr{G})$ fulfill the commutation relation

$$
\left[\varphi_{a}^{J}, \varphi_{b}^{k}\right]=\varphi_{[a, b]}^{j+k},
$$

i.e. they form a Kac-Moody type algebra [14]. As a particular case, if $a$ and $b$ belong to an Abelian subalgebra of $\mathscr{G}$, the corresponding vector fields are commuting symmetries of $N$,

$$
\left[\varphi_{a}^{j}, \varphi_{b}^{k}\right]=0, \quad L_{\varphi_{a}^{\prime}}(N)=0 .
$$

The algebra of these vector fields is said to be a Lenard bicomplex. An Abelian 
subalgebra can be easily constructed by means of a mapping $\Delta: \mathscr{G} \rightarrow \mathscr{G}$ which is itself a Nijenhuis tensor on $\mathscr{G}$

$$
[\Delta a, \Delta b]-\Delta[\Delta a, b]-\Delta[a, \Delta b]+\Delta^{2}[a, b]=0,
$$

and by an element $a_{0} \in \mathscr{G}$, which is a symmetry of $\Delta$

$$
\Delta\left[a_{0}, b\right]=\left[a_{0}, \Delta b\right] \quad(\forall b \in G),
$$

where $[a, b]$ is the commutator in $\mathscr{G}$. Then the Abelian subalgebra is given by

$$
a^{i}=\Delta^{i} a_{0} .
$$

The structure ( $\left.M, N, X, \Delta, a_{0}\right)$ is called a GN manifold (for more details, see [3] and references therein).

Remark 1. The involutive structure just introduced is independent of the existence of a Poisson structure in $M$, i.e. of a mapping $P: \mathscr{X}(M) \rightarrow \mathscr{X}^{*}(M)$ which is skew-symmetric and with vanishing Schouten bracket,

$$
\begin{aligned}
\langle\alpha, P \beta\rangle+\langle\beta, P \alpha\rangle=0 & \left(\alpha, \beta \in \mathscr{X}^{*}(M)\right), \\
\left\langle\alpha, L_{P \beta}(P) \gamma\right\rangle+\cdots+\cdots=0 & \left(\alpha, \beta, \gamma \in \mathscr{X}^{*}(M)\right) .
\end{aligned}
$$

Indeed, in most of the applications the manifold $M$ turns out to have an even richer structure, being bihamiltonian. This means that there is a Poisson tensor $P$ well-coupled (or compatible) with $N$, i.e. such that the mapping $Q=N P$ is itself a Poisson tensor.

Moreover, if the action of $\mathscr{G}$ is Poissonian, the vector fields of the Lenard bicomplex are themselves bihamiltonian, i.e. they are Hamiltonian with respect to both $P$ and $Q$.

Remark 2. An important example of GN manifold (suggested by the applications) is given by an affine hyperplane modelled on an associative algebra $A$ with unit and by a symmetry algebra $\mathscr{G}$ which is a subalgebra of $A: \mathscr{G} \subset A$ [3]. The starting symmetry $a_{0}$ and the Nijenhuis tensor $\Delta$ can be taken as the unit in $\mathscr{G}, a_{0}=1$, and as the right-multiplication by a suitable element $a \in G: \Delta b=R_{a} b=b a$ (the condition (1.6) is fulfilled since $A$ is an associative algebra, and the condition (1.7) is clearly trivial).

In the following Sects. 2 and 5 we will consider two GN structures defined on affine manifolds modelled on the same associative algebra.

\section{A First GN Manifold}

Let $A$ be an associative algebra with unit, $W \subset A$ a Lie subalgebra of $A$ and $V \subset W$ an associative subalgebra such that

$$
[V, W] \subset V
$$

and $c \in A-W$ a fixed element fulfilling the condition

$$
[c, W] \subset V, \quad\{c, V\} \subset V .
$$

Moreover, let $T: V \rightarrow W$ be a linear mapping fulfilling the modified Yang-Baxter 
(mYB) equation

$$
[T \varphi, T \psi]-T[\varphi, T \psi]-T[T \varphi, \psi]=-\lambda^{2}[\varphi, \psi] \quad(\varphi, \psi \in V)
$$

for some $\lambda \in \mathbb{C}$. As a base manifold, we consider the affine hyperplane

$$
M=V+\{c\}
$$

modelled on $V$, and we introduce in $M$ the tensor field $N$ defined by the linear mapping

$$
N_{u} \varphi=\lambda\{u, \varphi\}+[u, T \varphi] \quad(u \in M, \varphi \in V) .
$$

This mapping is well-defined on account of (i)-(ii) and fulfills the Nijenhuis condition (1.1) on account of (iii); so $M$ is a Nijenhuis manifold. Moreover, let us introduce the subalgebra $K \subset A$ of the "constants" defined as

$$
\begin{aligned}
K= & \{a \in A-W: a(W) \subset W,(W) a \subset W, a(V) \subset V,(V) a \subset V, \\
& T(a \varphi)=a T(\varphi), T(\varphi a)=T(\varphi) a, \forall \varphi \in V\},
\end{aligned}
$$

whose elements are consequently symmetries of $T: L_{a}(T)=0$, since, for any $\varphi \in V, L_{a}(T) \varphi=[a, T \varphi]-T[a, \varphi]=0$. As a symmetry algebra of $N$, we take the subalgebra $\mathscr{G} \subset K$ of the constants commuting with $c$,

$$
\mathscr{G}=\{a \in K:[a, c]=0\} .
$$

Indeed, the vector fields

$$
\varphi_{a}(u)=[u, a] \quad(a \in \mathscr{G}, u \in M)
$$

are well-defined and are symmetries of $N$ on account of (iv), (v); so $\mathscr{G}$ is a symmetry algebra of $N$. At least, the starting symmetry $a_{0}$ and the Nijenhuis tensor $\Delta$ in $\mathscr{G}$ will be taken as it has been already indicated in Remark 2 of Sect. 1.

Summarizing, we can state that

Proposition 2.1. The affine hyperplane $M$ is a $\mathrm{GN}$ manifold and the vector fields

$$
\varphi_{a}^{j k}=N^{j} X R_{a}^{k_{1}}
$$

define the Lenard bicomplex of $M$.

The construction of the GN manifold depends critically on the mYB condition (iii), which is indeed quite restrictive. An interesting example of a mapping $T$ fulfilling this condition can be obtained by considering a homomorphism $S$ of $A$ leaving both $W$ and $V$ invariant and such that $(1-S)$, restricted to $W$, be surjective onto $V$ and invertible

$$
S(V) \subset V \quad S(W) \subset W \quad W=(1-S)^{-1} V .
$$

Then the mapping $T: V \rightarrow W$ defined as

$$
T^{\varphi}=\lambda(1+S)(1-S)^{-1} \varphi \quad(\varphi \in V, \lambda \in \mathbb{C})
$$

fulfills the mYB condition (iii). Indeed, for $\varphi, \psi \in V$ it is $(\varphi=(1-S) \alpha, \psi=(1-S) \beta$, $\alpha, \beta \in W)$ 


$$
\begin{aligned}
{[T \varphi, T \psi]+\lambda^{2}[\varphi, \psi] } & =\lambda^{2}([(1+S) \alpha,(1+S) \beta]+[(1-S) \alpha,(1-S) \beta]) \\
& =2 \lambda^{2}(1+S)[\alpha, \beta], \\
T[T \varphi, \psi]+T[\varphi, T \psi] & =\lambda^{2} T([(1+S) \alpha,(1-S) \beta]+[(1-S) \alpha,(1+S) \beta]) \\
& =2 \lambda^{2} T(1-S)[\alpha, \beta]=2 \lambda^{2}(1+S)[\alpha, \beta] .
\end{aligned}
$$

Also the symmetry algebra of $N$ can be completely characterized in terms of $S$. Indeed, the assumptions (vi) entail that

$$
\left[(1+S),(1-S)^{-1}\right] \varphi=0 \quad(\varphi \in V),
$$

so that for any $a \in A-W$ such that

$$
(W) a \subset W \quad a(W) \subset W \quad V(a) \subset V \quad a(V) \subset V,
$$

the condition $S a=a$ is necessary and sufficient in order that

$$
T(\varphi a)=(T \varphi) a \quad T(a \varphi)=a(T \varphi) .
$$

This property can be easily checked by observing that for any $\varphi=(1-S) \alpha, \alpha \in W$, Eq. (2.8) entails the identity

$$
(1-S)(T(a \varphi)-a T(\varphi))=-2 \lambda(1-S) a \cdot S \alpha .
$$

Summarizing, the constants of this example are the fixed points of $S$, and the symmetry algebra is given by the fixed points of $S$ commuting with $c$. A realization of the corresponding GN manifold giving rise to the ILW hierarchy will be considered in the next Section.

Remark 1. If $A$ admits a Trace from $\operatorname{Tr}: A \rightarrow R$, we identify $V^{*}$ with $V$ by the pairing

$$
\langle\alpha, \varphi\rangle=\operatorname{Tr}(\alpha \varphi) \quad\left(\alpha \in V^{*}, \varphi \in V\right) .
$$

Then if $T$ is skew-symmetric with respect to (2.12), $M$ is a bihamiltonian manifold. Indeed, the tensor $P$ defined by

$$
P_{u} \alpha=[u, \alpha]
$$

is clearly a Poisson tensor and it is well-coupled with $N$, on account of the skew-symmetry of $T$ and of (iii). Moreover, the vector fields (2.3) are Hamiltonian with respect to $P$, on account of Jacobi's identity.

Remark 2. If it turns out that $T: V \rightarrow V$ and that $T^{2} \varphi=\lambda^{2} \varphi, T$ is itself a Nijenhuis tensor on account of (iii); in the following, we will consider an application where this condition is actually verified, furnishing the hierarchy of the Benjamin-Ono equation.

\section{The Reduction of the Lenard Bicomplex}

The vector fields (2.4) of the Lenard bicomplex do not directly correspond to integrable systems known from the literature. This is due to the conditions on the manifold $M$, the tensor $N$ and the symmetry algebra $\mathscr{G}$, which are rather restrictive. In particular, the conditions (ii) of Sect. 2 entail that $V$ be stable with respect to 
both left and right-multiplications by $c$, so that

$$
\sum_{k} v_{k} c^{k} \subset V \quad\left(v_{k} \subset V\right) .
$$

Thus if the order of $c$ is infinite, the dimension of $V$ cannot be finite and the evolution equations defined by the vector fields of the Lenard bicomplex give rise to a system in an infinite number of field functions. On the other hand, the search for invariant submanifolds of the Njenhuis tensor $N$ fails since, at the point $u=v+c$, $N$ takes the form

$$
N_{u} \varphi=\frac{1}{2}(\{v, \varphi\}+[c, \varphi]+2[v+c, T \varphi])+R_{c} \varphi
$$

(with the normalization choice $\lambda=1 / 2$ ). Due to the presence of the rightmultiplication $R_{c}$, the vector fields of the bicomplex cannot be tangent to any finite-dimensional submanifold (neither of order zero). Then, since the Nijenhuis tensor cannot be restricted, one looks for a reduction of the bicomplex, i.e. for suitable finite-dimensional submanifolds and for some special combinations of the fields of the bicomplex that are tangent to the submanifolds.

We now give a reduction theorem for the bicomplex constructed on a class of GN manifolds including that of Sect. 2. To this end, let us remark that the Nijenhuis tensor $N$ and the generator $X$ of the symmetry action of $\mathscr{G}$ are polynomial functions of the operators $L_{v}, R_{v}, \mathrm{ad}_{c}, L_{c}, R_{c}$ and $T$, so that we consider the following general form for $N$ and $X$ :

$$
\begin{aligned}
& N_{v+c}=p\left(L_{v}, R_{v}, \operatorname{ad}_{c}, L_{v} T, R_{v} T, \operatorname{ad}_{c} T\right)+R_{c}, \\
& X_{v+c}=q\left(L_{v}, R_{v}, \operatorname{ad}_{c}\right) .
\end{aligned}
$$

Under these assumptions, one can show that

Proposition 3.1. Let $M$ be an GN manifold modelled on an associative algebra with unit, defined by a Nijenhuis tensor (3.3), a symmetry generator (3.4), a symmetry algebra $\mathscr{G}$ given by (iv, v) of Sect. 2 and by $c \in \mathscr{G}$. If $Q \subset V$ is a subalgebra of $V$ fulfilling the conditions

$$
\operatorname{ad}_{c}(Q) \subset Q . \quad \operatorname{ad}_{c} T(Q) \subset Q, \quad T(Q) \cdot Q \subset Q, \quad Q \cdot T(Q) \subset Q,
$$

then the vector fields

$$
\varphi_{(n)}=\sum_{0}^{n}(-1)^{j}\left(\begin{array}{l}
n \\
j
\end{array}\right) N^{n-\jmath} X R_{c}^{\jmath 1}
$$

are tangent to the affine submanifold $M^{\prime}=Q+\{c\}$ modelled on $Q$.

Although the assumptions of this theorem are different from those of the reduction theorem which is proved in [3] for the KP case, on account of the peculiar properties of $T$, we omit its proof, since it can be performed in a quite similar way.

Explicitly, the first three vector fields of the form (3.6) are the following ones:

$$
\begin{aligned}
& \varphi_{(0)}=\varphi^{00}=0, \\
& \varphi_{(1)}=\varphi^{10}-\varphi^{01}=[q, c], \\
& \varphi_{(2)}=\varphi^{20}-2 \varphi^{11}+\varphi^{02}=\left[c, q^{2}\right]+2[q, T[c, q]]+2 T[c,[c, q]] .
\end{aligned}
$$




\section{A Realization of the GN Structure}

In this section we present a realization of the abstract scheme, with $T$ defined as in (2.5), giving rise to the hierarchy of the ILW equation. We make the following choices:

i) $\mathrm{A}$ is the algebra of differential operators in $\partial=\partial / \partial x$, whose coefficients are $C^{\infty}$ functions on the real axis

$$
f(x)=\sum_{k \geqq 0} f_{k}(x) \partial^{k}, \quad f_{k} \in C^{\infty}(\mathbb{R}, \mathbb{C})
$$

ii) $W$ is Lie subalgebra of differential operators with coefficients taking asymptotically opposite constant values for $|x| \rightarrow \infty$,

$$
w(x)=\sum_{k \geqq 0} w_{k}(x) \partial^{k} \quad w_{k}( \pm \infty)=a_{k} \quad\left(a_{k} \in \mathbb{C}\right) .
$$

iii) $V$ is the subalgebra of differential operators with rapidly vanishing coefficients for $|x| \rightarrow \infty$,

$$
\varphi(x)=\sum_{k \geqq 0} \varphi_{k}(x) \partial^{k}, \quad \varphi_{k} \in \mathscr{S}(\mathbb{R}, \mathbb{C})
$$

iv) The constant $c$ fixing the affine hyperplane $M$ is equal to $\partial$ so that for any $f \in A$,

$$
[c, f]=\sum_{k \geqq 0} f_{k x} \partial^{k} \quad\left(f_{k x}=\partial f_{k} / \partial x\right) .
$$

v) The algebra homomorphism $S$ is the adjoint action by $g:=\exp (\eta \partial)$

$$
S \alpha:=\operatorname{Ad}_{\exp (\eta \hat{c})^{x}} \quad(\eta \in \mathbb{C})
$$

With these choices, the conditions (i), (ii) of Sect. 2 are clearly fulfilled. Moreover, the equivariance property of the adjoint action entails that

$$
S=\exp \left(\eta \operatorname{ad}_{\hat{c}}\right)
$$

So, the effect of $S$ on $f \in A$ is merely a shift

$$
S\left(\sum_{k \geqq 0} f_{k}(x) \partial^{k}\right)=\sum_{k \geqq 0} f_{k}(x+\eta) \partial^{k},
$$

and the fixed points of $S$ are the differential operators with constant coefficients. The symmetry algebra $\mathscr{G}$ is formed by $a \in A$

$$
a=\sum_{k \geqq 0} a_{k} \partial^{k} \quad\left(a_{k x}=0\right)
$$

Then, on account of Proposition 1 of $[7]$, for $\eta=2 i \delta(\delta \in \mathbb{R})$ and for any $\varphi \in V$, there is a unique $w \in W$ related with $\varphi$ by

$$
w(x)=i(1-S)^{-1} \varphi(x)=\frac{i}{2} \varphi(x)+i(T \varphi)(x),
$$

where (by (iv) of Sect. 2) $T$ acts on $\varphi$ as

$$
T \varphi=\sum_{k \geqq 0}\left(T \varphi_{k}\right) \hat{\partial}^{k},
$$


and $\left(T \varphi_{k}\right)(x)$ is the well-known integral transform

$$
\left(T \varphi_{k}\right)(x)=\frac{-i}{8 \delta} P \int \operatorname{Coth} \frac{\pi(x-\xi)}{2 \delta} \varphi_{k}(\xi) \mathrm{d} \xi,
$$

$P$ denoting the Cauchy's principal value.

Now we are able to easily construct the Lenard bicomplex (2.4) for this realization. However, we are more interested in the reduction on a submanifold $M^{\prime}$ fulfilling the conditions of the Proposition 3.1. In this regard, it can be easily checked that the subalgebra of zero-order differential operators fulfills these conditions. So, at any point

$$
u \in M^{\prime}: u=q+\hat{\partial}, \quad(q \in \mathscr{S}(\mathbb{R}, \mathbb{C}))
$$

the vector fields (3.7) are realized by

$$
\begin{aligned}
& \varphi_{(1)}=-q_{x}, \quad \varphi_{(2)}=2 q q_{x}+2 T q_{x x}, \\
& \varphi_{(3)}=\frac{1}{4} q_{x x x}+3 q^{2} q_{x}+3 T\left(q q_{x}\right)_{x}+3\left(q T q_{x}\right)_{x}+3 T^{2} q_{x x x} .
\end{aligned}
$$

They are the first fields of the ILW hierarchy in $1+1$ dimensions [9].

Remark 1. The abstract scheme suggests in a rather natural way a formal extension of this realization to the case of two spatial dimensions. Indeed, if one considers the algebra $V$ of differential operators in $\partial_{x}$ and $\partial_{y}$, whose coefficients are Schwartz functions in $\mathbb{R}^{2}$, one could repeat the previous realization by taking $g=\exp \left(\eta \partial_{y}\right)$, replacing the integral transform (4.11) by the integral transform $\bar{T}$ acting on the $y$ variable

$$
\left(\bar{T} \varphi_{k}\right)(x, y)=\frac{-i}{8 \delta} P \int \operatorname{Coth} \frac{\pi}{2 \delta}(y-\xi) \varphi_{k}(x, \xi) \mathrm{d} \xi .
$$

Then the structure of the vector fields (4.13) remains formally the same, giving rise to the so-called ILW hierarchy in $2+1$ dimensions [15].

Remark 2. As it is known, by performing the limit $\delta \rightarrow \infty$ the integral transform (4.11) tends to the Hilbert transform $H$. In this case, one can choose $V=W$, so that $H^{2} \varphi=\lambda^{2} \varphi$; so, $H$ becomes a Nijenhuis tensor and Eqs. (4.13) give the Benjamin-Ono hierarchy. On the other hand, the limit $\delta \rightarrow 0$ gives the hierarchy of KP in the so-called second representation [9].

Remark 3. Another realization of the previous abstract bicomplex can be performed by taking the algebra of distributional kernels of the form

$$
f\left(x_{1}, x_{2}\right)=\sum_{k \geqq 0} f_{k}\left(x_{1}, x_{2}\right) \delta^{k}\left(x_{1}-x_{2}\right)
$$

where $\delta^{k}\left(x_{1}-x_{2}\right)=\lambda^{k} \delta\left(x_{1}-x_{2}\right) / \partial x_{1}^{k}$ and $\delta\left(x_{1}-x_{2}\right)$ is Dirac's distribution. By so doing, the ILW hierarchy is obtained in the framework of the extended formalism [9]. 


\section{Another GN Manifold}

Let us now consider another GN structure. It is defined on the same affine manifold previously introduced in Sect. 2, only with the difference that the conditions (ii) are replaced by the weaker condition

$$
[c, W] \subset V .
$$

This new structure is based on the existence of a linear mapping $\widetilde{T}: V \rightarrow W$ fulfilling the Yang-Baxter (YB) equation

$$
[\tilde{T} \varphi, \tilde{T} \psi]=\tilde{T}[\tilde{T} \varphi, \psi]+\tilde{T}[\varphi, \tilde{T} \psi] \quad(\varphi, \psi \in V)
$$

instead of the mYB condition (iii) of Sect. 2. So, the GN structure in $M$ is given by the same algebra $K$ of the constants defined by (iv) of Sect. 2 (with $T$ replaced by $\widetilde{T}$ ) and by the same symmetry generators (2.3). Instead, the Nijenhuis tensor is now given by the "chiral" tensor

$$
N_{u} \varphi=[u, \widetilde{T} \varphi]
$$

Remark 1. As it is well-known, if $\tilde{T}$ is invertible the inverse map $D=\widetilde{T}^{-1}$ is derivation on the Lie subalgebra $W \subset A$. On the other hand, if $D$ is an invertible derivation, then $\widetilde{T}=D^{-1}$ fulfills (iii'). In this case, the constants previously defined in Sect. 2 are actually the elements of the kernel of $D$, and the GN manifold takes the same structure as for the chiral representation of KP systems [3]. An interesting example of a GN manifold corresponding to a solution of (iii') is obtained by considering a suitable element $g \in A$. It is assumed to be invertible, fulfilling the stability conditions with respect to $V$ and $W$ and such that $\operatorname{ad}_{g}$, restricted to $W$, be surjective onto $V$ and invertible. Then it follows that its adjoint action $S=L_{g} R_{g}^{-1}$ fulfills all the conditions for the homomorphism of the algebra as in Sect. 2, and that the mapping

$$
\tilde{T}: V \rightarrow W, \quad \widetilde{T} \varphi:=R_{g}^{-1}(1-S)^{-1} \varphi
$$

is a solution of the YB equation (iii'). Indeed, its inverse map $D$ is an invertible derivation

$$
D \alpha=\tilde{T}^{-1} \alpha=-\operatorname{ad}_{g} \alpha .
$$

As for the symmetry algebra, it can be shown that also in this case the subalgebra $K$ defined by ((iv), Sect. 2) is given by the fixed points of $S$ and that $g$ itself is a constant.

Remark 2. Although the construction of this section may seen to be formally a particular case of the one of Sect. 2, corresponding to a solution of (iii) with $\lambda=0$, it is interesting on its own, since the particular solution (5.2) has altogether different properties with respect to $T$. Consequently, the reduction of the Lenard bicomplex must be performed by a completely different technique, as will be shown in the next section.

Remark 3. If a Trace-form is given making $\tilde{T}$ skew-symmetric, $\tilde{T}$ is itself a 
presymplectic tensor compatible with $N$, since $N=P \widetilde{T}$, with the Poisson tensor $P$ given by (2.13). The affine manifold $M$ is also in this case a bihamiltonian manifold.

\section{A New Reduction Theorem for the Lenard Bicomplex}

In this section we shall discuss the reduction of the bicomplex corresponding to the particular solution (5.2) of the Yang-Baxter equation. We recall that in order to reduce the Lenard bicomplex one has to determine a subalgebra $Q \subset V$ and a family of vector fields of the bicomplex which are tangent to the affine manifold $M^{\prime}=Q+\{c\}$.

Since at any point $u \in M$ the Nijenhuis tensor (5.1) takes the form

$$
N_{v+c}=\left(L_{v}-R_{v}+\mathrm{ad}_{c}\right) \tilde{T},
$$

it does not belong to the class (3.3) and the reduction theorem (3.1) cannot be applied. To overcome this difficulty, we take advantage of the freedom provided by the bicomplex scheme, i.e. the choice of the Nijenhuis tensor $\Delta$ on the symmetry algebra. So, in this case we will consider the bicomplex corresponding to the choice

$$
\Delta=R_{g}
$$

(where $g \in \mathscr{G}$ is the invertible element appearing in the definition of $\tilde{T}$ ) instead of $\Delta=R_{c}$ is in the previous reduction theorem. Then one can prove that

Proposition 6.1. Let $M$ be a GN manifold modelled on an associative algebra with unit, defined by the Nijenhuis tensor $N$,

$$
N_{v+c}=\left(L_{v}-R_{v}+\operatorname{ad}_{c}\right) \tilde{T} \quad \tilde{T}=R_{g}^{2-1}(1-S)^{-1} \quad(u=v+c \in M),
$$

the symmetry generator

$$
X_{v+c}=L_{v}-R_{v}+\mathrm{ad}_{c},
$$

and by the symmetry algebra $\mathscr{G}$ given by (iv, v) Sect. 2, with $c \in \mathcal{G}$. If $Q \subset V$ is any subalgebra of $V$ fulfilling the conditions

$$
\begin{gathered}
S(Q) \subset Q \quad Q \cdot(1-S)^{-1} Q \subset Q \quad(1-S)^{-1} Q \cdot Q \subset Q, \\
{\left[c,(1-S)^{-1} Q\right] \subset Q,}
\end{gathered}
$$

then the vector fields

$$
\varphi_{(n)}:=N^{n} X R_{g}^{n_{1}}
$$

are tangent to the affine manifold $M^{\prime}=Q+\{c\}$ modelled on $Q$.

The reduction theorem follows from the following lemmas:

Lemma I. The vector fields $\varphi^{o n}=X \Delta^{n}$ a can be written as

$$
\varphi^{o n}=R_{g}^{n}[v, a]_{n},
$$

where $[v, a]_{n}:=v a-a S^{n} v$.

From the definition of $X$, it follows that

$$
\varphi^{o n}=\left[v+c, \Delta^{n} a\right]=\left[v, a g^{n}\right]=\left(v a-a g^{n} v g^{-n}\right) g^{n}=R_{g}^{n}[v, a]_{n} .
$$


Lemma II. The mapping $G_{i}$ defined as

$$
G_{i} \varphi:=\left[u,(1-S)^{-1} \varphi\right]_{i} \quad(i=0,1, \ldots ; \varphi \in Q)
$$

fulfill the relations

$$
\begin{aligned}
R_{g}^{-1} G_{i} R_{g} & =G_{i+1}, \\
R_{g}^{-1}\left(\prod_{0}^{k} G_{i}\right) R_{g} & =\prod_{1}^{k+1} G_{i} .
\end{aligned}
$$

The first relation follows directly from the definition, since for any integer $k$ it is

$$
\begin{aligned}
R_{g}^{-1} G_{k} R_{g} \varphi & =R_{g}^{-1}\left[u,(1-S)^{-1} R_{g} \varphi\right]_{k}=\left(u \cdot(1-S)^{-1} \varphi g-(1-S)^{-1} \varphi g \cdot S^{k} u\right) g^{-1} \\
& =u \cdot(1-S)^{-1} \varphi-(1-S)^{-1} \varphi \cdot S^{k+1} u=G_{k+1} \varphi .
\end{aligned}
$$

The second property coincides with the first one for $k=0$; then it can be proved by induction, since it is

$$
\begin{aligned}
R_{g}^{-1}\left(\prod_{0}^{k+1} G_{i}\right) R_{g} & =\left(R_{g}^{-1} \cdot \prod_{0}^{k} G_{i} \cdot R_{g}\right)\left(R_{g}^{-1} G_{k+1} R_{g}\right) \\
& =\prod_{1}^{k+1} G_{i} \cdot G_{k+2}=\prod_{1}^{k+2} G_{i} .
\end{aligned}
$$

At last, one can prove that

Lemma III. $\quad N^{n} R_{g}^{n}=\prod_{0}^{n-1} G_{i}$.

Since the property holds for $n=1$,

$$
N R_{g} \varphi=\left[u,(1-S)^{-1} \varphi\right]=G_{0} \varphi,
$$

it can be proved by induction

$$
\begin{aligned}
N^{n+1} R_{g}^{n+1} & =N\left(N^{n} R_{g}^{n}\right) R_{g}=G_{0} R_{g}^{-1} \cdot \prod_{0}^{n-1} G_{i} \cdot R_{g} \\
& =G_{0} \prod_{1}^{n} G_{i}=\prod_{0}^{n} G_{i} .
\end{aligned}
$$

On account of the previous lemmas, the vector fields $\varphi_{(n)}$ defined by (6.7) take the form

$$
\begin{aligned}
\varphi_{(n)} & =N^{n} X R_{g}^{n_{1}} \\
& \stackrel{(\mathrm{I})}{=} N^{n} R_{g}^{n}[v, 1]_{n} \\
& \stackrel{(\mathrm{III})}{=}\left(\prod_{0}^{n-1} G_{i}\right)\left(1-S^{n}\right) v .
\end{aligned}
$$

So, the reduction of the bicomplex is straightforward, since $Q$ is stable with respect to $S$ and to $G_{i}$ on account of the assumptions (6.5) and (6.6). 
The vector fields of the reduced bicomplex are then obtained by the following scheme:

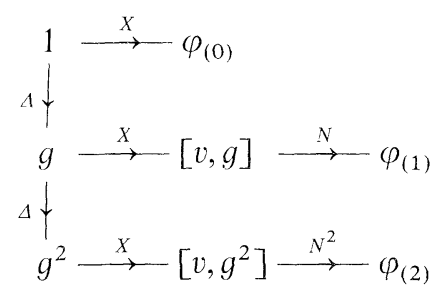

At any point $u=q+c$ of the reduction submanifold $M^{\prime}$ one obtains

$$
\begin{aligned}
\varphi_{(0)}= & 0, \\
\varphi_{(1)}= & {[q, \tilde{T}[q, g]]+[c, \tilde{T}[q, g]], } \\
\varphi_{(2)}= & {\left[q, \tilde{T}\left[q, \tilde{T}\left[q, g^{2}\right]\right]\right]+\left[q, \tilde{T}\left[c, \tilde{T}\left[q, g^{2}\right]\right]\right] } \\
& +\left[c, \tilde{T}\left[q, \widetilde{T}\left[q, g^{2}\right]\right]\right]+\left[c, \tilde{T}\left[c, \tilde{T}\left[q, g^{2}\right]\right]\right] .
\end{aligned}
$$

A realization of this abstract scheme can be performed as well as in Sect. 4. In particular, if one considers the algebra of differential operators, with the same choices for $c, g$ as in Sect. 4, one can easily check that the subalgebra $Q$ of Schwartz functions on the line (i.e. differential operators of zero order) fulfills the conditions (6.5), (6.6) of the reduction theorem.

We end this section by observing that taking into account the definitions of $T$ and $\widetilde{T}$,

and that

$$
T=\frac{1}{2}(1+S)(1-S)^{-1} ; \quad \widetilde{T}=R_{g-1}(1-S)^{-1} ; \quad S=\operatorname{Ad}_{g}
$$

$$
[\tilde{T} \varphi, g]=\varphi \quad\{\tilde{T} \varphi, g\}=2 T \varphi \quad(\varphi \in V) .
$$

A direct inspection allows one to conclude that the vector fields (6.18) are exactly the vector fields (3.7), so that the hierarchies corresponding to $T$ and $\tilde{T}$ actually coincide (a proof of this fact, for the extended formalism referred to in Remark 3 of Sect. 4, has been given in [9]).

\section{Concluding Remarks}

In this paper we have shown how the algebraic approach of the GN manifold and the Lenard bicomplex successfully explains the recursive properties of the integrodifferential ILW hierarchy of NEE's, as well as those of the differential NEE's considered elsewhere. In this regard, we point out that the integro-differential operators (4.11), (4.14) appear explicitly only in the last step of the construction, i.e. in the realization by a particular algebra, whereas the reduced vector fields (3.7) are defined in any abstract algebra in terms of any solution of the mYB equation. So different solutions can give rise to new integrable NEE's. The abstract approach enables one to obtain the recursion scheme of a NEE both in local and in extended form [2], i.e. both in the algebra of differential operators and in the algebra of distributional kernels. Moreover, it allows one to handle in a unitary way with 
NEE's both in one and in two spatial dimensions, the difference being given only by the choice of the algebra and of some parameters (e.g., $c=\partial_{x}, g=\exp \eta \partial_{x}$ for the ILW in $(1+1)$ and $c=\partial_{x}, g=\exp \eta \partial_{y}$ for the ILW in $(2+1)$ dimensions). At last, the existence of the second representation for the ILW hierarchy (as well as for KP systems) gives rise to the following two problems:

i) does any integrable NEE admit two (or more) representations?

ii) how are the different reduction techniques (such as those given by Theorems (3.1), (6.1)) related with these representations?

To the best of our knowledge, they are still open problems, which we hope to be able to analyze in a forthcoming paper.

Acknowledgements. Many helpful discussions with F. Magri are gratefully acknowledged. This work has been partially supported by the Italian Ministry of Public Education and by the G.N.F.M. of the Italian C.N.R.

\section{References}

1. Konopelchenko, B. G.: Non-linear integrable equations. Lecture Notes in Physics, vol. 270. Berlin, Heidelberg, New York: Springer 1987

2. Fokas, A. S.: Stud. Appl. Math. 77, 253 (1987)

3. Magri, F., Morosi, C., Tondo, G.: Nijenhuis G-manifolds and Lenard bicomplexes: A new approach to KP systems. Commun. Math. Phys. 115, 457 (1988)

4. Fokas, A.S., Fuchssteiner, B.: Phys. Lett. 86A, 341 (1981)

5. Kodama, Y., Satsuma, J., Ablowitz, M.J.: Phys. Rev. Lett. 46, 687 (1981)

6. Gibbon, H., Kupershmidt, B : Phys. Lett. 79A, 31 (1980)

\%. Lebedev, D. R., Radul, A.O.: Generalized Internal Long Waves equatıons: Constructıon, Hamiltonian structure and conservation laws. Commun. Math. Phys. 91, 543 (1983)

8. Fokas, A. S., Santini, P. M.: Bi-Hamiltonian formulations of the Kadomtsev-Petviashvili and Benjamin-Ono equations. J. Math. Phys. 29, 604 (1988).

9. Santini, P. M.: Bi-Hamiltonian formulations of the Intermediate Long Wave equation. J. Math. Phys. (1988)

10. Semenov-Tian-Shansky, M. A.: Funct. Anal. Appl. 17, 259 (1983)

11. Oevel, W.: R-structures, Yang-Baxter equations and related involution theorems. J. Math. Phys. (1988)

12. Magri, F., Morosi, C., Tondo, G.: The geometry of soliton equations. In Proceedings of the I.S.A.M., Paipa C. I. F., Levi, D., Winternitz, P. (eds.), pp. 146-187. Singapore: World Scientific 1988

13. Magri, F., Morosi, C., Tondo, G.: In: Non-linear evolutions. Leon, J. JP. (ed.), pp. 231-244. Singapore; World Scientific 1988

14. Goddard, P., Olive, D.: Int. J. Mod. Phys. A1, 303 (1986)

15. Santini, P. M.: Dimensional deformations of integrable systems: An approach to integrability in multidimension. I. Preprint 586, Università di Roma (1988)

Communicated by A. Jaffe

Received July 29, 1988; in revised form November 7, 1988 
